\title{
Langerhans Cell Histiocytosis of the Temporal Bone
}

\author{
Daniel Thomas Ginat ${ }^{1} \cdot$ Daniel N. Johnson ${ }^{2} \cdot$ Nicole A. Cipriani $^{2}$
}

Received: 3 April 2015/Accepted: 16 April 2015/Published online: 24 April 2015

(C) Springer Science+Business Media New York 2015

\begin{abstract}
Langerhans cell histiocytosis involving the temporal bone region is uncommon and can resemble malignant neoplasms on imaging due to high cellularity. Although recognizing the presence of sharp margins with beveled-edges can be helpful, tissue sampling is often necessary for confirming the diagnosis. Cytology classically demonstrates kidney-bean shaped nuclei within the Langerhans cells and immunohistochemical staining is positive for S-100, peanut agglutinin (PNA), MHC class II, CD1a, and Langerin (CD 207). These features are exemplified in this sine qua non radiology-pathology correlation article.
\end{abstract}

Keywords Langerhans cell histiocytosis - Temporal bone $\cdot$ CT $\cdot$ MRI $\cdot$ Pathology $\cdot$ Cytology

\section{Case Description}

The patient is a 10-month-old male with no significant past medical history who presented with a palpable mass in the left preauricular region. The patient was afebrile and examination revealed a white polypoid mass in the left ear.

Daniel Thomas Ginat

dtg1@uchicago.edu; ginatd01@gmail.com

1 Department of Radiology, Pritzker School of Medicine, University of Chicago, $5841 \mathrm{~S}$ Maryland Avenue, Chicago, IL 60637, USA

2 Department of Pathology, Pritzker School of Medicine, University of Chicago, Chicago, IL, USA

\section{Radiographic Features}

Temporal bone CT (Fig. 1) and MRI with contrast (Fig. 2) were obtained. The CT showed an enhancing soft tissue mass in the left lateral skull base, predominantly involving the tympanic, mastoid, and squamosal portions of the temporal bone. In particular, the lesion demonstrated sharply defined margins with "beveled-edges". The corresponding MRI showed an avidly enhancing T2 hypointense mass, suggestive of a hypercellular process. The MRI also showed mild protrusion of the mass into the underlying epidural space and extension into overlying subcutaneous tissues, where there was suggestion of mild inflammatory changes. However, there was no associated cerebral edema.

\section{Diagnosis}

Histopathology of the temporal bone lesion biopsy specimen demonstrated dense sheets of monotonous cells underlying squamous epithelium. The cells displayed moderate amounts of eosinophilic cytoplasm and kidney bean-shaped or folded nuclei, typical of Langerhans cell histiocytosis (previously known as eosinophilic granuloma or Histiocytosis X). Scattered multinucleated giant cells and eosinophils were also present. The lesional histiocytes stained positive for CD1a, S-100, and Langerin (Fig. 3).

\section{Treatment}

The patient was started on a protocol consisting of a 12 month course of combined intravenous vinblastine $\left(6 \mathrm{mg} / \mathrm{m}^{2}\right)$ and prednisolone $\left(40 \mathrm{mg} / \mathrm{m}^{2}\right)$, which the patient tolerated well. 
Fig. 1 Axial CT image in the soft tissue window, a shows a soft tissue mass centered in the left temporal bone (arrow). The coronal CT image in the bone window, $\mathbf{b}$ shows that the lesion has well-defined margins with beveled-edges (arrowheads)

Fig. 2 Axial fat-suppressed T2weighted (a) and coronal

(b) fat-suppressed post-contrast T1-weighted MR images show a $\mathrm{T} 2$ hypointense avidly

enhancing mass centered in the left lateral skull base with epidural and dermal extension
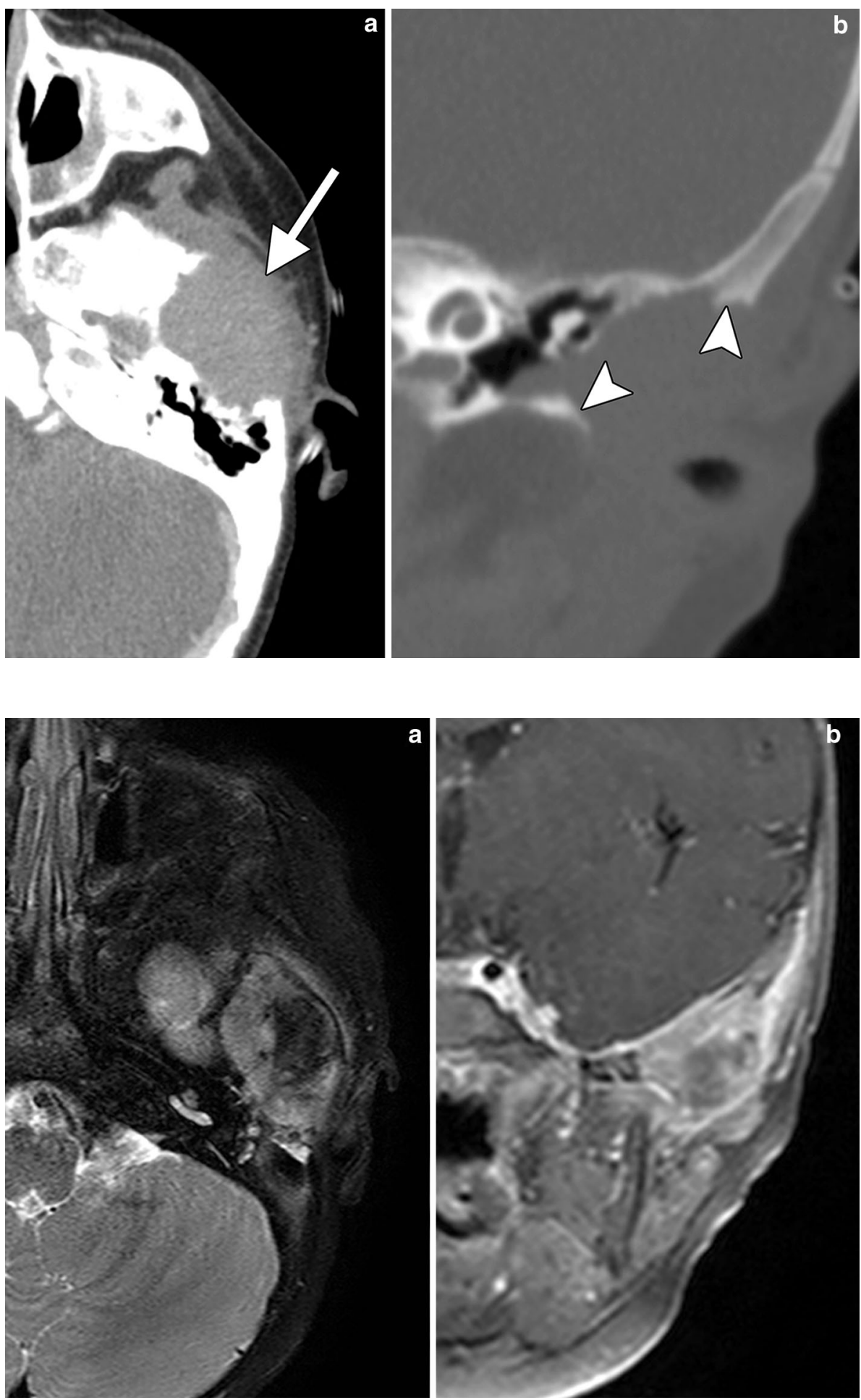
Fig. 3 Hematoxylin and eosin stained photomicrograph image obtained at $\times 60$ original magnification (a) shows numerous Langerhans cells with kidney-shaped nuclei, admixed with eosinophils. Immunohistochemistry photomicrograph obtained at $\times 60$ original magnification shows positive staining for Langerin (b)

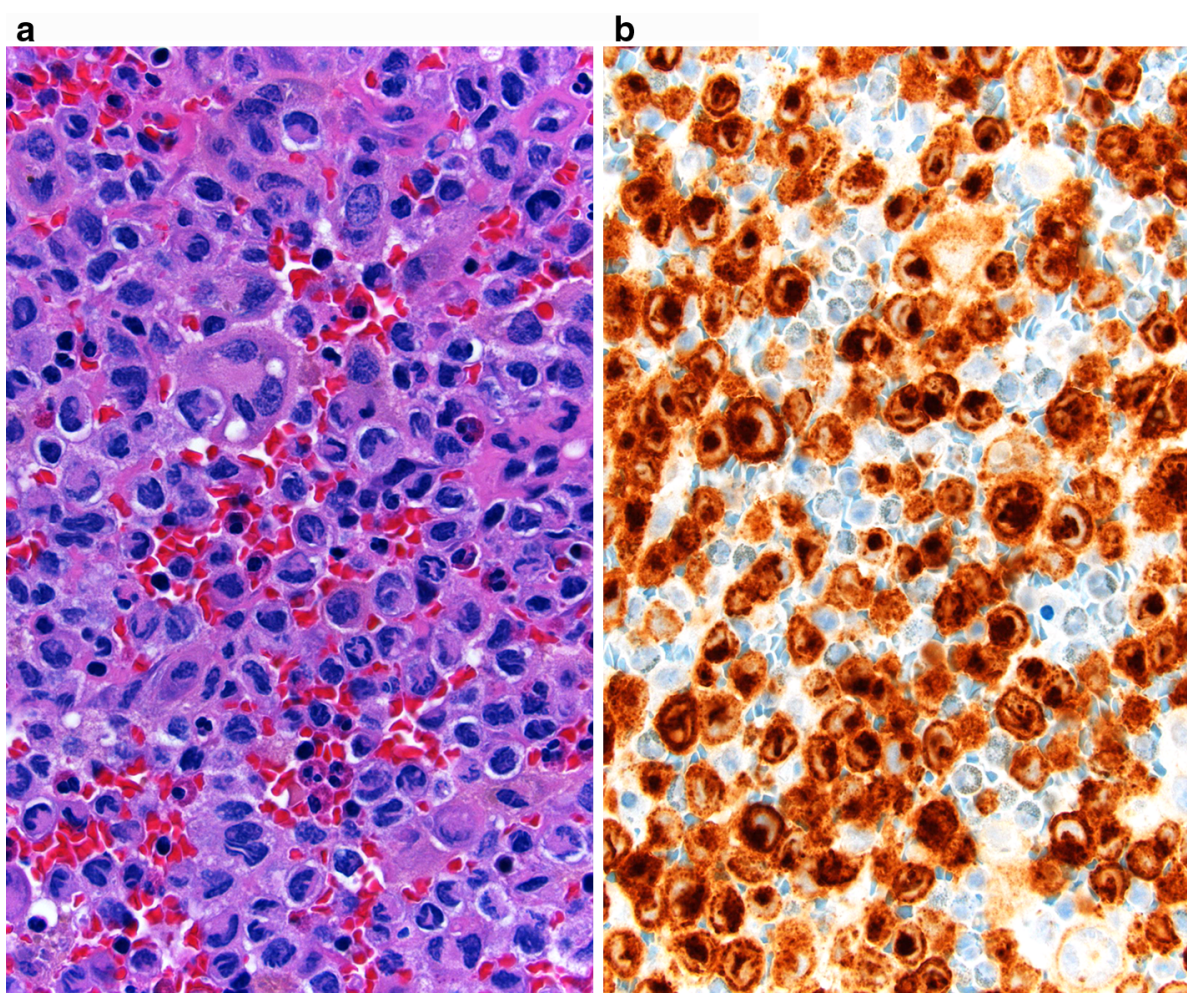

MRI obtained 6 months after starting therapy demonstrated resolution of left temporal bone mass.

\section{Discussion}

Langerhans cell histiocytosis is an uncommon condition related to the abnormal proliferation of Langerhans cells, which are macrophages that are normally present only in the dermis. Up to approximately $60 \%$ of patients with Langerhans' cell histiocytosis have otologic involvement as an isolated manifestation of the disease or as part of systemic disease [1]. The symptoms associated with Langerhans cell histiocytosis of the temporal bone region may include sensorineural or conductive hearing loss, otalgia, otorhea, and/or an external auditory canal polyp or mass, as in this case [1].

MRI and CT are complimentary modalities for evaluating pediatric temporal bone region masses [2]. On MRI, Langerhans cell histiocystosis typically appear as enhancing masses with low T2 signal [3]. The lesions can mimic malignant neoplasms, such as lymphoma, rhabdomyosarcoma, and neuroblastoma metastases, particularly in the presence of low diffusivity on diffusion-weighted MRI due to high cellularity [4]. On CT, Langerhans cell histiocytosis typically appear as lytic lesions with sharp 'beveled-edges' associated with a soft tissue mass, but may otherwise appear nonspecific, potentially mimicking an infectious process, for instance [3]. Skeletal surveys and abdominal ultrasonography can be useful for assessing the presence of systemic disease. Indeed, this patient also had abdominal lymphadenopathy and partial collapse of the T8 vertebral body associated with Langerhans cell histiocytosis.

Recent observation that Langerhans cell histiocytosis is a clonal process with activating BRAF mutations suggests that LCH is a neoplastic condition [6]. Clinical diagnosis can be challenging and tissue sampling via fine needle aspiration or biopsy may be required. The histologic appearance of Langerhans cell histiocytosis is variable, but it often appears as cellular sheets of Langerhans cells admixed with a polymorphous population of eosinophils, neutrophils, and lymphocytes. Rare plasma cells can also be present. The diagnosis relies on identifying sheets of Langerhans cells, which classically appear as large cells with abundant eosinophilic cytoplasm and characteristically large, irregular, and grooved nuclei resembling kidneys or coffee beans [5]. Multinucleated Langerhans cells can also be present, as in this case. Fine needle aspiration cytology can also be useful for confirming the presence of Langerhans cells particularly if a cell block is used for immunohistochemical confirmation. The cells stain positively for S-100, peanut agglutinin (PNA), MHC class II, CD1a, and Langerin (CD 207) [5].

Treatment options for temporal bone Langerhans cell histiocytosis include surgery, radiotherapy, chemotherapy, 
and steroids [7]. Successfully treated lesions in the temporal bone region typically regress over the course of 1 year and the affected bone is reconstituted [3].

Conflict of interest None.

\section{References}

1. Saliba I, Sidani K, El Fata F, Arcand P, Quintal MC, Abela A. Langerhans' cell histiocytosis of the temporal bone in children. Int J Pediatr Otorhinolaryngol. 2008;72:775-86.

2. Stern JS, Ginat DT, Nicholas JL, Ryan ME. Imaging of pediatric head and neck masses. Otolaryngol Clin North Am. 2015;48: 225-46.
3. Fernández-Latorre F, Menor-Serrano F, Alonso-Charterina S, Arenas-Jiménez J. Langerhans' cell histiocytosis of the temporal bone in pediatric patients: imaging and follow-up. AJR Am J Roentgenol. 2000;174:217-21.

4. Ginat DT, Mangla R, Yeaney G, Johnson M, Ekholm S. Diffusionweighted imaging for differentiating benign from malignant skull lesions and correlation with cell density. AJR Am J Roentgenol. 2012;198:W597-601.

5. Chandekar SA, Shah VB, Kavishwar V. Cytological diagnosis of Langerhans cell histiocytosis with cutaneous involvement. J Cytol. 2013;30:81-3.

6. Badalian-Very G, Vergilio JA, Fleming M, Rollins BJ. Pathogenesis of Langerhans cell histiocytosis. Annu Rev Pathol. 2013;8:1-20.

7. Yildirim-Baylan M, Cureoglu S, Paparella MM. Langerhans' cell histiocytosis of the temporal bone. Otol Neurotol. 2012;33:e15-6. 